\title{
Log-periodic modulation to power law of change in the geoelectric potential difference observed on Niijima Island prior to volcanic and seismic activation in the Izu island region, Japan 2000
}

\author{
By Haruo TANAKA \\ Earthquake Prediction Research Center, Tokai University, 3-20-1, Orido, Shimizu, Shizuoka 424-8610 \\ (Communicated by Seiya UYEDA, M. J. A., Nov. 12, 2002)
}

\begin{abstract}
Anomalous changes in the geoelectric potential difference between electrodes on Niijima Island first appeared at the end of April 2000 about two months before the onset of a major volcanic and seismic activity in the Izu island region. They ceased at the end of August 2000 simultaneously with the decline of the volcanic and seismic activity. The temporal change in the magnitude of $0.01 \mathrm{~Hz}$ Fourier coefficient of the geoelectric potential difference was analyzed on the basis of a critical "time-to-failure" model. It was found that the temporal change was well explained by a power-law decorated with a log-periodic oscillation. This indicates that the possible generation mechanism of the electric potential changes was related to a critical phenomenon. Several possible mechanisms that may cause this phenomenon are suggested.
\end{abstract}

Key words: Log-periodic oscillation; geoelectric potential; hierarchical structure; failure; discrete scale invariance; critical phenomenon.

Introduction. Recently, failures in heterogeneous systems have been extensively studied. ${ }^{1)^{-9)}}$ Some of these studies have shown that failures are considered as critical phenomena, i.e., there is a quantity that exhibits a diverging rate when the critical stress is approached. Moreover, it has been shown that quantities, such as acoustic emissions, ${ }^{5), 6)}$ the total number of broken bonds, ${ }^{7)}$ and the cumulative Benioff strain release ${ }^{8), 9)}$ can be expressed by power laws with logperiodic modulations as a function of the stress $\sigma=\sigma(\mathrm{t})$ in the vicinity of their critical point as in eq. [1],

$$
\mathrm{D}=\mathrm{A}+\mathrm{B}\left(\sigma_{\mathrm{c}}-\sigma\right)^{-\xi}\left(1+\mathrm{C} \cos \left[2 \pi \mathrm{f} \log \left(\sigma_{\mathrm{c}}-\sigma\right)+\phi\right]\right),[1]
$$

where $\sigma_{c}$ is the critical stress and A,B,C, $\xi$, f and $\phi$ are parameters. If we assume $\sigma(\mathrm{t})=$ at (a is constant) and the critical stress is reached when $t=t_{f}$, eq. [1] is rewritten as

$$
\mathrm{D}=\mathrm{A}+\mathrm{B}^{\prime}\left(1-\frac{\mathrm{t}}{\mathrm{t}_{\mathrm{f}}}\right)^{-\xi}\left(1+\mathrm{C} \cos \left[2 \pi \mathrm{f} \log \left(1-\frac{\mathrm{t}}{\mathrm{t}_{\mathrm{f}}}\right)+\phi^{\prime}\right]\right),[2]
$$

where $\mathrm{B}^{\prime}=\mathrm{B}\left(\mathrm{a} / \mathrm{t}_{\mathrm{f}}\right)^{-\xi}$ and $\phi^{\prime}=\phi+2 \pi \mathrm{f} \log \mathrm{a}-2 \pi \mathrm{f} \log \mathrm{t}_{\mathrm{f}}$. This equation indicates two important features of the quantity D, i.e., (1) predictability of the divergence and (2) the punctuated growth. The feature (1) means that we can predict, in advance, through the failure time $t_{f}$, when the systems will destroy. The feature (2) originates from the log-periodic oscillation superposed on the power law. In the following, let us consider the origin of the log-periodic oscillation following refs. 9) and 13). The log-periodic oscillation superposed on the power law is considered to be the manifestation of the discrete scale invariance in the vicinity of a critical point. The scale invariance of an observable $\mathrm{O}(\mathrm{x})$ is in general defined by

$$
\mathrm{O}(\mathrm{x})=\lambda^{\beta} \mathrm{O}(\lambda \mathrm{x})
$$

for a scale change $x \rightarrow \lambda x$. The solution of eq. [3] is

$$
\mathrm{O}(\mathrm{x})=\mathrm{Cx}^{\alpha}
$$

where $\mathrm{C}$ is constant. If we allow the complex exponent $\alpha$, it should satisfy the relation

$$
(\alpha+\beta) \log \lambda=2 \pi \text { in (n is arbitrary integer), }
$$

where the identity $1=\mathrm{e}^{2 \pi \mathrm{in}}$ is used. In Euclidian systems, defined by the positive integer dimensions, we can take arbitrary positive real value of $\lambda$. Therefore, eq. [5a] should hold for arbitrary positive real value of $\lambda$. This means $\alpha+\beta=0$ and $\mathrm{n}=0$. Thus the exponent is real $\left(\alpha=-\beta=-\frac{\log \mu}{\log \lambda}\right)$. In discrete fractal systems, $\lambda$ can be written as $\lambda=\mathrm{a}^{\mathrm{m}}$, where $\mathrm{a}$ is constant and $\mathrm{m}$ is an arbitrary positive integer. Using this condition, eq. [5a] becomes 


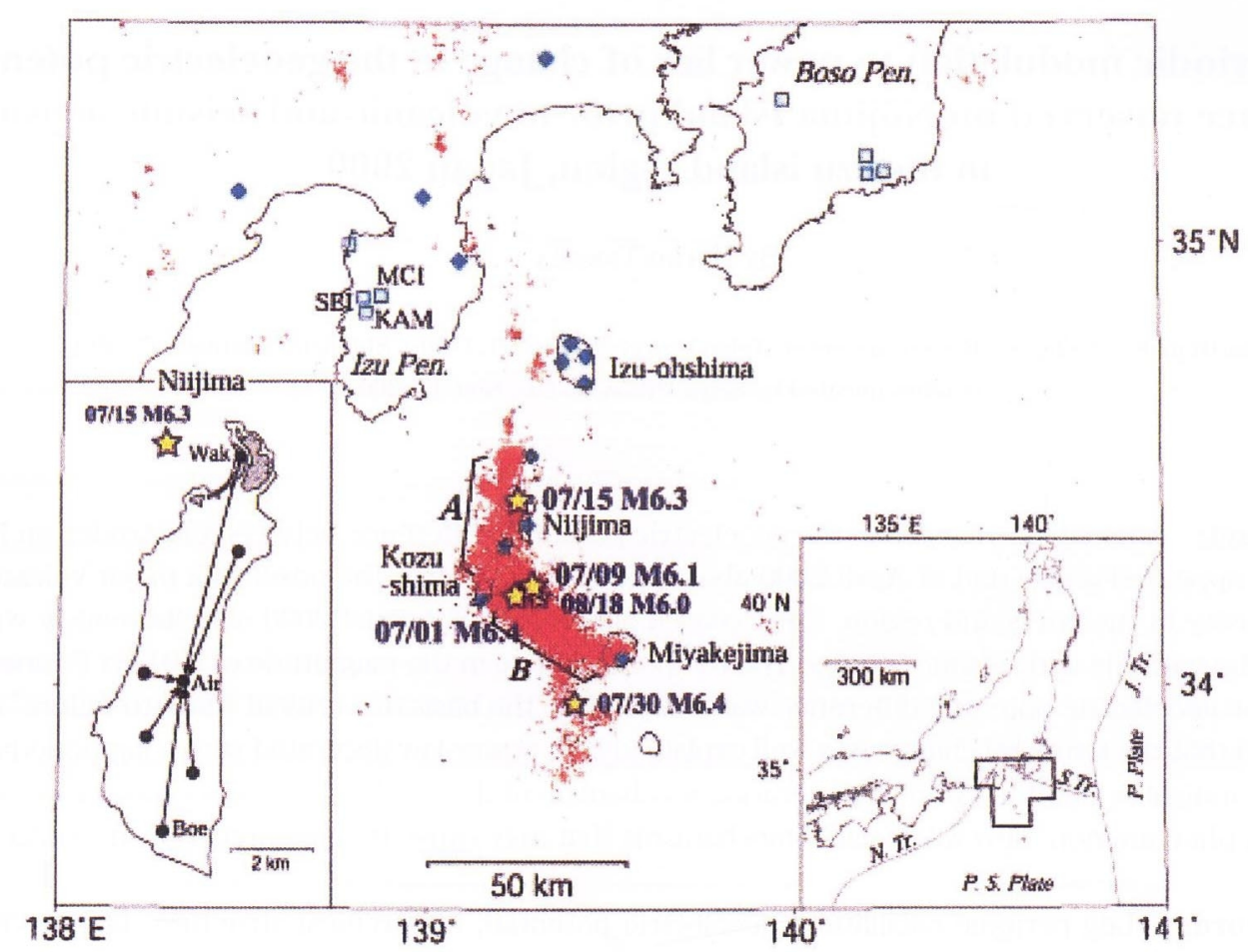

Fig. 1. Index map of the Izu island region. ${ }^{9)}$ Red dots are $M \geqq 0$ earthquakes according to JMA (Japan Meteorological Agency) for June 1 - September 30, 2000. Yellow stars are $M \geqq 6$ earthquakes. Diamonds: geoelectric stations. Squares: geomagnetic stations. Lower right inset is a map of Japan with plate boundaries. P. Plate: Pacific Plate, P. S. Plate: Philippine Sea Plate, N. Tr.: Nankai Trough, S. Tr.: Sagami Trough, J. Tr.: Japan Trench. Inset in the left shows the long dipole configuration of Niijima station.

$(\alpha+\beta) \mathrm{m} \log \mathrm{a}=2 \pi \mathrm{in}$.

Eq. [5b] holds for an arbitrary positive integer $m$ if we put $\mathrm{n}=\mathrm{mk}$. The exponent can then be written as

$$
\alpha=-\beta+2 \pi \mathrm{i} \frac{\mathrm{k}}{\log \mathrm{a}}=-\beta+2 \pi \mathrm{i} \frac{\mathrm{n}}{\log \lambda} .
$$

Substituting eq. [5c] into eq. [4], $\mathrm{O}(\mathrm{x})$ becomes

$$
\mathrm{O}(\mathrm{x})=\operatorname{CRe}\left[\sum_{\mathrm{a}_{\mathrm{n}} \mathrm{x}^{\alpha \mathrm{n}}}\right]=\mathrm{Cx}^{-\beta}\left[\mathrm{a}_{0}+2 \mathrm{a}_{1} \cos \left(2 \pi \frac{\log \mathrm{x}}{\log \lambda}\right)\right] \cdot[6]
$$

where $a_{-1}=a_{1}$ is used to ensure $O(x)$ to be real. Eq. [6] indicates that the observable $\mathrm{O}(\mathrm{x})$ is invariant over the discrete scale changes as $x \rightarrow \lambda^{n} x$. The apparent identity of eq. [6] and eq. [2], guarantees that the quantity D is invariant over the discrete scale changes of the distance from the failure time $t_{\mathrm{f}}$. Now, it is clear that the feature (2), the punctuated growth of the quantity D, originates from its discrete scale invariance in the vicinity of the failure time.

In this paper, the temporal change in the magnitude of $0.01 \mathrm{~Hz}$ Fourier coefficient of the geoelectric potential difference between electrodes observed on Niijima Island prior to the volcanic and seismic activity in the Izu island region in the summer of $2000^{10)-11)}$ is studied on the basis of eq. [2]. Within the author's knowledge, any electromagnetic field variations prior to large earthquakes have not been analyzed so far from the viewpoint of critical "time-to-failure". It may shed a new light on finding the possible generation mechanism of the preseismic temporal changes in geoelectric potential difference, of which existence is now fairly certain despite some controversy. ${ }^{12)}$

Analysis of data. We had a geoelectric and geomagnetic monitoring system in the Izu island region when the 2000 volcanic and seismic activity started (Fig. 1). The results of observation relevant to the present study ${ }^{10)}$ were as follows: On Nijima Island, there was a geoelectric potential monitoring station with 6 long $(1 \sim 6 \mathrm{~km})$ and 6 short (about $30 \mathrm{~m})$ dipoles using telephone cables. The sampling rate was once per $10 \mathrm{~s}$. From about two months prior to the onset of the volcanic and seismic activity, the long dipole connecting Air (Airport) and Wak (Wakago Village) and the short dipole at Wak started to show visually clear innumerable unusual changes. Similar changes were not observed on any other dipoles at stations in the region. 


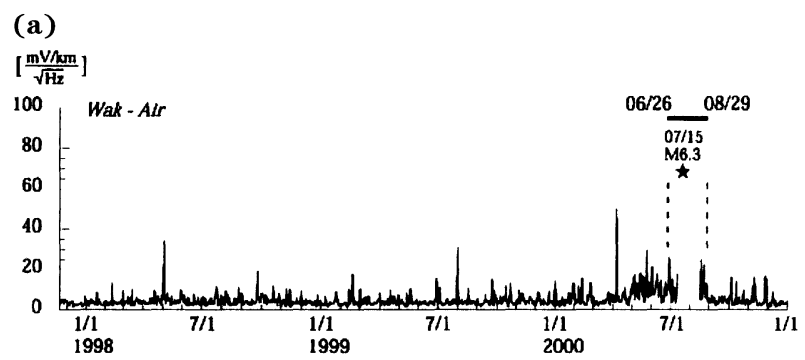

(b)

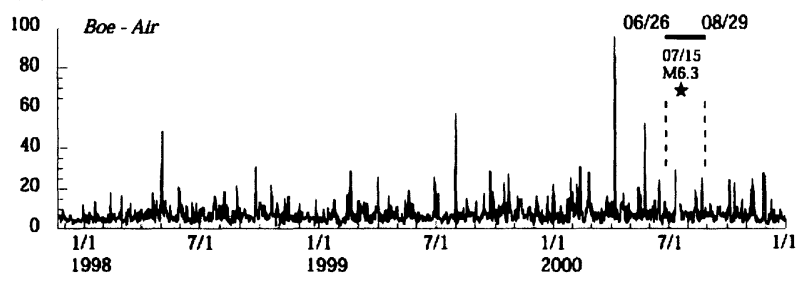

Fig. 2. Three year record of the magnitude of $0.01 \pm 0.0003 \mathrm{~Hz}$ Fourier coefficient of geoelectric field. ${ }^{9)}$ (a) : Wak-Air dipole, (b): Boe-Air dipole. The lack of data for Wak-Air dipole in the year 2000 was due to power failure and mechanical damage caused by a typhoon and the July 15 M6.3 earthquake (star) of which epicenter was close to Wakago Village (Wak in Fig. 1). The volcanoseismic activity started on June 26, 2000 and lasted to August 29 as shown by the vertical dotted line.

Fig. 2(a) and (b) show the three-year records of the magnitude of Fourier coefficient at $0.01 \pm 0.0003 \mathrm{~Hz}$ band of the geoelectric potential difference over Wak-Air dipole and Boe-Air dipole. Fourier coefficient was calculated each day using one-day data. Some rise of the magnitude of the Fourier coefficient from the end of April, 2000 may be noticed in Wak-Air data. Changes common to Wak-Air and Boe-Air dipoles are mainly due to geomagnetic field variations originating in the upper atmosphere. In order to eliminate these noises, the ratio

$\mathrm{Q}_{\mathrm{obs}}(\mathrm{j})=\frac{\mathrm{F}_{\text {wak }}^{(\mathrm{j})}(\mathrm{f})}{\mathrm{F}_{\text {boe }}^{(\mathrm{j})}(\mathrm{f})}$ of these two-time series data was taken as shown in Fig. 3, where $F_{\text {wak }}^{(i)}(f)$ and $F_{\text {boe }}^{(i)}(f)$ are the quantities shown in Fig. 2(a) and (b), i.e., the magnitudes of j-th day's $0.01 \mathrm{~Hz}$ Fourier coefficients of geoelectric potential difference at Wak-Air dipole and Boe-Air dipole, respectively. The increase before the volcanic and seismic activity can now be more clearly seen. The same tendency was verified for the frequency range of $0.0001 \sim 0.03 \mathrm{~Hz}$, but the enhancement was strongest in $0.006 \sim 0.03 \mathrm{~Hz}$ range. This is the reason why $0.01 \mathrm{~Hz}-$ band was chosen for this study. The quantity $Q_{o b s}(n)$ is reminiscent of the emission rate of acoustic emissions in rock failure experiments, ${ }^{5)}$ suggesting eq. [2] would be applicable to the present case also.

As $Q_{o b s}(n)$, which we call the emission rate here-

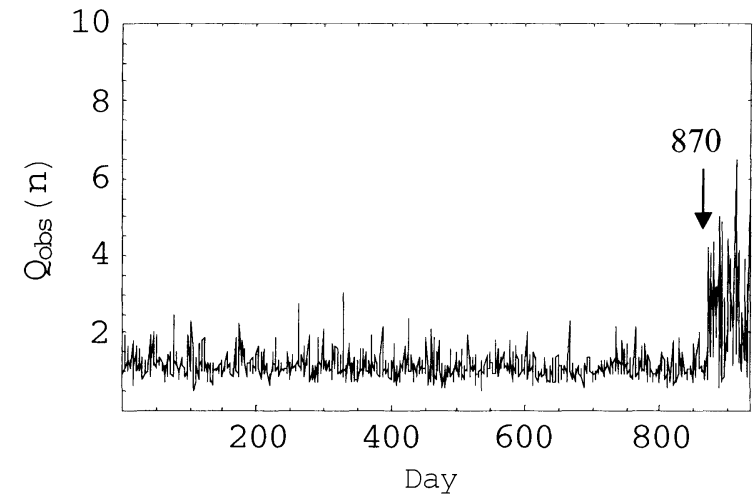

Fig. 3. The daily change in the emission rate $Q_{\text {obs }}(j)=\frac{F_{\text {wak }}^{(j)}(f)}{F_{\text {bre }}^{(j)}(f)}$ from $\mathrm{n}=1$ (November 21,1997$)$ to $\mathrm{n}=934$ (June 30, 2000). The emission rate is accelerated at $n=870$ (April 25, 2000).

after, is fluctuating, it is difficult to estimate when the system has entered a "critical" state. In order to make a better estimation of the onset of the critical state, it is convenient to take the cumulative emission $\mathrm{I}_{\mathrm{obs}}(\mathrm{n})$, i.e., the sum of $Q_{o b s}(n)$ from the first day of observation through the n-th day as

$$
I_{\text {obs }}(n)=\sum_{j=1}^{n} Q_{o b s}(j) .
$$

This is considered to be identical to operating a low pass filter. By analogy with the case of acoustic emission, ${ }^{5),(6)}$ it was examined whether or not $I_{o b s}(n)$ is modeled by,

$I(n)=C_{1}\left(1-\frac{n}{t_{f}}\right)^{-\xi}\left(1+C_{2} \cos \left[\eta \log \left[1-\frac{n}{t_{f}}\right]+\phi\right]\right)+A_{1} n+A_{2},[8]$

where $t_{\mathrm{f}}$ indicates the day of "catastrophe". The second term in the right-hand side $A_{1} n$ is added for taking into account the secular trend of $I_{o b s}(n)$. The optimization of the parameters is made by the least square method. Namely, a set of parameters $\left(\mathrm{C}_{1}^{(0)}, \mathrm{C}_{2}^{(0)}, \xi^{(0)}, \eta^{(0)}, \phi^{(0)}, \mathrm{A}_{1}^{(0)}\right.$, $\left.A_{2}^{(0)}, t_{f}^{(0)}\right)$ which gives the minimum value of error defined by

$$
\begin{aligned}
& \Delta\left(\mathrm{C}_{1}, \mathrm{C}_{2}, \xi, \eta, \phi, \mathrm{A}_{1}, \mathrm{~A}_{2}, \mathrm{t}_{\mathrm{f}}\right)= \\
& \frac{1}{\mathrm{n} \max -\mathrm{n} \min +1} \sum_{\mathrm{n}=\mathrm{n} \min }^{\mathrm{n} \max }\left(\mathrm{I}(\mathrm{n})-\mathrm{I}_{\text {obs }}(\mathrm{n})\right)^{2},
\end{aligned}
$$

was determined. The way to determine $\mathrm{n} \max$ and $\mathrm{n}$ min is described below. Moreover, we plotted $\delta\left(\mathrm{t}_{\mathrm{f}}\right)$ defined by

$$
\delta\left(\mathrm{t}_{\mathrm{f}}\right)=\Delta\left(\mathrm{C}_{1}^{(0)}, \mathrm{C}_{2}^{(0)}, \xi^{(0)}, \eta^{(0)}, \phi^{(0)}, \mathrm{A}_{1}^{(0)}, \mathrm{A}_{2}^{(0)}, \mathrm{t}_{\mathrm{f}}\right),
$$

as a function of $t_{\mathrm{f}}$ in order to estimate the day of "cata- 


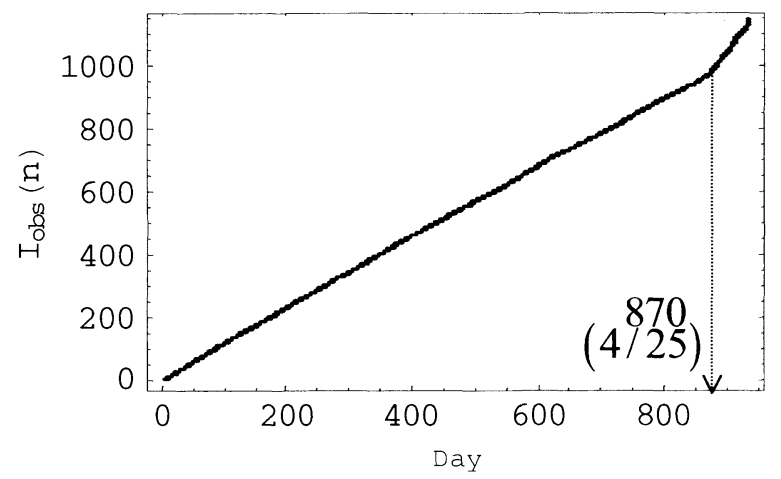

Fig. 4. The daily change in the cumulative emission $I_{\text {obs }}$ from the day $n=1$ (November 21, 1997) to the day $n=934$ (June 30, 2000). Before the day $n=870$ (April 25, 2000), $I_{\text {obs }}$ linearly increases and the deviation from the straight line is very small. After the day $n=870, I_{\text {obs }}$ increases with larger fluctuation.

Table I. The most suitable parameters for the cumulative emission

\begin{tabular}{llllllll}
\hline $\mathrm{C}_{1}^{(0)}$ & $\mathrm{C}_{2}^{(0)}$ & $\xi^{(0)}$ & $\eta^{(0)}$ & $\phi^{(0)}$ & $\mathrm{A}_{1}^{(0)}$ & $\mathrm{A}_{2}^{(0)}$ & $\mathrm{t}_{\mathrm{f}}^{(0)}$ \\
\hline 1.85 & 0.04 & 0.66 & 12.79 & -1.80 & 1.11 & 48.8 & 940 \\
\hline
\end{tabular}

strophe". If $\delta\left(\mathrm{t}_{\mathrm{f}}\right)$ has a deep minimum, we can predict the accurate day of catastrophe $t_{\mathrm{f}}^{(0)}$.

Results and discussion. In Fig. $4, \mathrm{I}_{\mathrm{obs}}(\mathrm{n})$ from $\mathrm{n}=1$ (November 27,1997$)$ to $\mathrm{n}=934$ (June 30,2000$)$ is shown to have two distinct segments separated by the day $n=870$ (April 25, 2000). The segment before April 25 is considered to be the secular trend around the observation site. Thus, the parameter $A_{1}$ is determined as the slope of $\mathrm{I}_{\mathrm{obs}}(\mathrm{n})$ before April 25. The segment after April 25 indicates that the area around the observation site entered an "unusual state". It seems that this state was closely related to the preparation process of the volcanic and seismic activity starting on the day June 26. But all the period of this unusual state may not necessarily be a critical state. So, a care was taken in assigning the upper limit $\mathrm{n} \max$ and lower limit $\mathrm{n}$ min in eq. [8] because eq. [8] should hold only in the vicinity of a critical point, i.e., in the critical state. The upper limit could be simply set to $n \max =929$ (June 25), because the day $n=929$ was the closest day to the catastrophe and the last day that was free from the direct influence of possible changes caused by the volcanic and seismic activity. On the other hand, the lower was determined as follows: We first plot $\log \left|I_{o b s}(n)-A_{1}^{(0)} n-A_{2}\right|$ vs. $\log \left[1-\frac{n}{t_{f}}\right]$ by changing two parameters $t_{\mathrm{f}}$ and $\mathrm{A}_{2}$. If the system is in a critical state, the log-log plot should be approximated by a straight-line segment and the abscissa of both ends of it
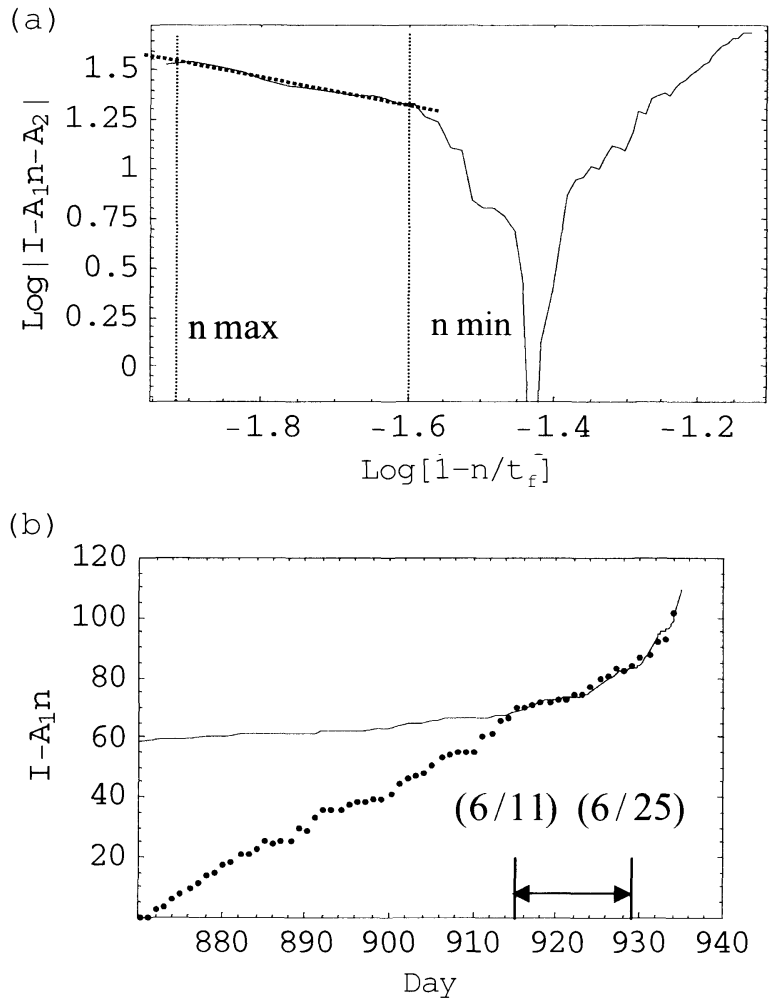

Fig. 5. (a) $\log \left|I_{\text {obs }}(n)-A_{1}^{(0)} n-A_{2}^{(0)}\right|$ is plotted as a function of $\log [1$ $\left.-\frac{n}{t_{\mathrm{f}}}\right]$ for $\mathrm{t}_{\mathrm{f}}=940$ and $\mathrm{A}_{2}^{(0)}=48.8$. In the region, $-1.93<\log \left[1-\frac{\mathrm{n}}{940}\right]$ $<-1.6$, i.e., $929>\mathrm{n}>915$ (from June 11 to June 25), the log-log plot is approximated by a straight line. (b) $I_{o b s}(n)-A_{1}^{(0)} n$ (dots) and $\mathrm{I}(\mathrm{n})-\mathrm{A}_{1}^{(())} \mathrm{n}$ (solid curve) with the parameters (Table I) are plotted as a function of $n$ from the day $n=870$ (April 25) to the day $\mathrm{n}=934$ (June 30 ). The coincidence of the data points and the fitting curve in the region $n>915$ (after June 11) is good. An arrow shows the period of data used for parameter fitting.

should coincide $n$ min and $n$ max (see Fig. 5(a)). Thus, we can estimate $n$ min when two parameters $\mathrm{t}_{\mathrm{f}}$ and $\mathrm{A}_{2}$ are given. We are now ready for the least square parameter fitting of eq. [9].

The calculated parameters for the cumulative emission are listed in Table I. In Fig. 5 (a), the log-log plot is shown using the optimal values of $t_{\mathrm{f}}=940$ (July 6 ) and $\mathrm{A}_{2}^{(0)}=48.8$. We can see in the region $-1.93<\log \left[1-\frac{n}{940}\right]<-1.6$, i.e., $\mathrm{n} \max =929>\mathrm{n}>\mathrm{n} \min =915$ (from June 11 to June 29), the log-log plot is actually well approximated by a straight-line segment with a slope about 0.6 . From this fact, it is presumed that the system has entered a critical state from June 11. In Fig. 5 (b), $\mathrm{I}_{\mathrm{obs}}(\mathrm{n})-\mathrm{A}_{1}^{(0)} \mathrm{n}(\mathrm{dots})$ and $I(n)-A_{1}^{(0)} n$ (solid curve) with the parameters (Table I) as a function of $\mathrm{n}$ are shown. The coincidence of the data 
Table II. The most suitable parameters for the emission rate

\begin{tabular}{llllllll}
\hline $\mathrm{C}_{1}^{(0)}$ & $\mathrm{C}_{2}^{(0)}$ & $\xi^{(0)}$ & $\eta^{(0)}$ & $\phi^{(0)}$ & $\mathrm{A}_{1}^{(0)}$ & $\mathrm{A}_{2}^{(0)}$ & $\mathrm{t}_{\mathrm{f}}^{(0)}$ \\
\hline 0.14 & 0.54 & 0.68 & 10.5 & 5.8 & 1.11 & 0.13 & 940 \\
\hline
\end{tabular}

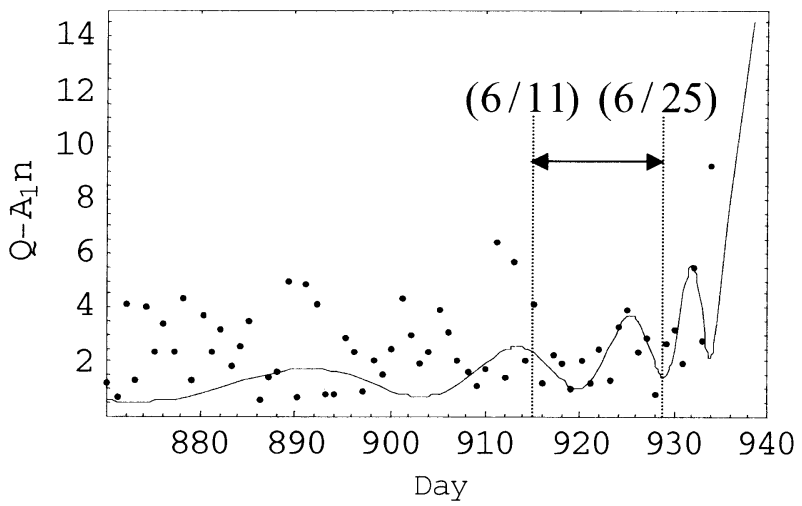

Fig. 6. $Q_{o b s}(n)-A_{1}^{(0)} n\left(\right.$ dots) and $Q(n)-A_{1}^{(0)} n$ (solid curve) with the parameters (Table II) are plotted as a function of $n$ from the day $\mathrm{n}=870$ (April 25) to the day $\mathrm{n}=934$ (June 30). The coincidence of the data points and the fitting curve in the region from the day $\mathrm{n}=915$ (June 11 ) to the day $\mathrm{n}=933$ (June 29) is good. After the day $n=934$ (June 30 ), there is no coincidence due to the volcanic and seismic activity that has already started on June 26. An arrow shows the period of data used for parameter fitting.

points and the fitting curve is good in the range from $\mathrm{n}=915$ (June 11) to $\mathrm{n}=929$ (June 25). Moreover, the coincidence of the data points and the fitting curve is also good in the range from $n=930$ (June 26) to $n=934$ (June 30) although the data during this period were not taken into account for the parameter fitting. These two facts indicate that the anomalous changes from June 11 to June 30 were probably the manifestation of a critical phenomenon. On the other hand, as the anomalous changes from $n=870$ (April 25) to $n=914$ (June 10) did not fit to eq. [8] with the parameters in Table I, the mechanism of their generation was probably not related to critical phenomena. Taking cumulative quantity smoothens the noise and provides the higher signal-overnoise ratio. However, it significantly reduces the feature of log-periodic oscillations presented in $Q_{o b s}(n)$. In order to demonstrate the log-periodic oscillations more clearly, it may be better to analyze the emission rate. We performed the parameter fitting for the emission rate using the same $n$ max and $n$ min as above. The optimal parameters are listed in Table II. In Fig. $6, Q_{o b s}(n)-A_{1}^{(0)} n$ (dots) and $Q(n)-A_{1}^{(0)} n$ (solid curve) with the parameters in Table II, are shown as a function of $n$. The coincidence of the data points and the fitting curve from $n=$ 915 (June 11) to $n=929$ (June 25) is good. Moreover,

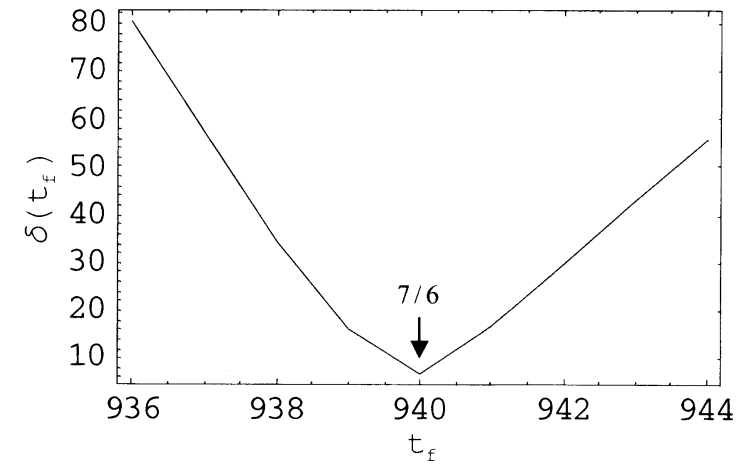

Fig. 7. $\delta\left(\mathrm{t}_{\mathrm{f}}\right)$ of eq. [10] for the cumulative emission is plotted as a function of $\mathrm{t}_{\mathrm{f}} . \delta\left(\mathrm{t}_{\mathrm{f}}\right)$ reaches its minimum at $\mathrm{t}_{\mathrm{f}}=940$. Thus, the catastrophe is presumed to be $n=940$ (July 6).

the coincidence of them from $n=929$ (June 25) to $n=933$ (June 30) is also good despite the data during this period was not used for the reason explained above. The last data point $n=934$ (June 30) is not on the fitting curve. This may, however, be affected by the volcanic and seismic activity that had already started on June 26.

In Fig. $7, \delta\left(\mathrm{t}_{\mathrm{f}}\right)$ of eq. [10] for the cumulative emission is plotted as a function of $\mathrm{t}_{\mathrm{f}}$. There is a minimum at $\mathrm{n}=940$ (July 6), which may be taken as the best predicted date for the catastrophe. The volcanic activity of Miyakejima started on June 26, and the first M6.4 earthquake in the area occurred on July 1 and the M6.3 earthquake occurred on July 15 in the very vicinity of Niijima (Fig. 1).

Anomalous geomagnetic field changes were also observed in Izu Peninsula (Fig. 1). ${ }^{10)}$ In ref. 10), the time series of the square root of eigenvalues corresponding to the third principal components is shown to have increased from about three months prior to the volcanic and seismic activity. We made a similar analysis on the above time series to see if similar critical behavior could be obtained. However, no clear critical behavior was confirmed. Since the daily fluctuations of the eigenvalues of the third principal components were large, critical behavior may have been hidden in them even if existed.

We have seen that the anomalous change of the geoelectric potential difference obeyed the log-periodic oscillation superposed on the power law, i.e., it is invariant over the discrete scale changes of the distance from the catastrophe day. Let us now consider its possible generation mechanisms. The geoelectric potential difference can be a function of many different quantities. Here, we consider four types of quantities that may play a crucial role in generating it through various specific 
processes, such as electrokinetic, piezoelectric, motion of charged dislocations or charged impurities, etc. The first type is the quantities related to hierarchical systems. According to the previous studies on such systems, ${ }^{13)-17)}$ the quantities that characterize failure or magnetization exhibit the discrete scale invariance. The second type is the strain energy accumulated under a constant strain rate in heterogeneous systems. According to ref. 18), due to the dynamic interplay between the heterogeneities of a system and the stress field, the accumulated strain energy becomes discrete scale invariant. The third of type is related to diffusion of water in the crust. Studies of diffusion of particles in heterogeneous structures under external forces ${ }^{19), 20)}$ showed that $\alpha(\mathrm{t})=\frac{\mathrm{d} \log \left\langle\mathrm{R}^{2}(\mathrm{t})\right\rangle}{\mathrm{d} \log \mathrm{t}}$ exhibits log-periodic oscillations, where $\left\langle R^{2}(t)\right\rangle$ is the mean square displacements of the particles at time $t$. If water in the upper crust diffuses due to a pressure caused by a thin sheeted dike that intruded between Miyakejima and Kozu-shima, ${ }^{21,22)}$ the generated electric potential may also show the log-periodic oscillations. The fourth type is the number of states in non-linear dissipative systems with a small degree of freedoms ${ }^{23), 24)}$ which also shows log-periodic oscillations superposed on power laws in the vicinity of an attractor. If the governing equations of the preparation process of the volcanic and seismic activity are found to belong to the same universality class as those of the non-linear dissipation system with a small degree of freedoms, this concept may become applicable to the type of problem of our interest. The equations of motion taking into account the rate and state dependent friction formulation ${ }^{25), 26)}$ may belong to such a universality class.

Conclusion. The anomalous geoelectric potential changes between some electrodes on Niijima Island started at the end of April 2000, about two months before the onset (June 26) of the major volcanic and seismic activity in the Izu island region. The cumulative emission as well as the emission rate from June 11 to June 25 was found to obey a power law with a log-periodic modulation. This fact suggests that the geoelectric potential changes precursory to the 2000 activity in the Izu island region was closely related to a critical phenomenon in which signal emission maintains the scale invariance over the discrete scale change of the distance from the failure day. Several possible mechanisms that may cause this phenomenon are suggested.

Acknowledgements. The author thanks Dr.
M. Kamogawa for helping the development of the computer program and Dr. K. Hattori for providing the author with the geomagnetic field data, and acknowledges Dr. S. Uyeda, M. J. A., for constructive discussions and critical reading.

\section{References}

1) Zapperi, S., Ray, P., Stanley, H. E., and Vespignani, A. (1999) Phys. Rev. E 59, 5049-5057.

2) Shnirman, M. G., and Blanter, E. M. (1998) Phys. Rev. Lett. 81, 5445-5448.

3) Hergarten, S., and Neugebauer, H. J. (2000) Phys. Rev. E 61, 2382-2385.

4) Andersen, J. V., Sornette, D., and Leung, K. (1997) Phys. Rev. Lett. 78, 2140-2143.

5) Sobolev, G. A., and Tyupkin, Yu. S. (2000) Izv. Physics of the Solid Earth 36, 138-149.

6) Johansen, A., and Sornette, D. (2000) Eur. Phys. J. B 18, 163-181.

7) Newman, W. I., Turcotte, D. L., and Gabrielov, A. D. (1995) Phys. Rev. E 52, 4827-4835.

8) Anifrani, J. C., Le Foc'h, C., Sornette, D., and Souillard, B. (1995) J. Phys. I (Paris) 5, 631-638.

9) Sornette, D. (1998) Phys. Reports 297, 239-270.

10) Uyeda, S., Hayakawa, M., Nagao, T., Molchanov, O., Hattori, K., Orihara, Y., Gotoh, K., Akinaga, Y., and Tanaka, H. (2002) Proc. Natl. Acad. Sci. U. S. A. 99, 7352-7355.

11) Japan Meteorological Agency (2000) Earth Planets Space $\mathbf{5 2}$ $1-8$.

12) Geller, R. (ed.) (1996) Geophys. Res. Lett. 23, 2025-2028.

13) Sornette, D. (2000) Critical Phenomena in Natural Science, Springer, Berlin, pp. 131-135, 267-278.

14) Turcotte, D. L., Smalley, R. F., and Solla, S. A. (1985) Nature 313, 671-672; Smalley, R. F., Turcotte, D. L., and Solla, S. A. (1985) J. Geophys. Res. 90, 1894-1900.

15) Saleur, H., Sammis, C. G., and Sornette, D. (1996) J. Geophys. Res. 101, 17661-17667.

16) Lessa, J. C., and Andrade, R. F. S. (2000) Phys. Rev. E 62, 3083-3089

17) Andrade, R. F. S. (2000) Phys. Rev. E 61, 7196-7199.

18) Sahimi, M., and Arbabi, S. (1996) Phys. Rev. Lett. 77, 3689-3692.

19) Saadatfar, M., and Sahimi, M. (2002) Phys. Rev. E 65, 036116-1-036116-8.

20) Stauffer, D., and Sornette, D. (1998) Physica A 252, 271-277.

21) Toda, S., Stein, R., and Sagiya, T. (2002) Nature 419, 58-61.

22) Kodaira, S., Uhira, K., Tsuru, T., Sugioka, H., Suyehiro, K., and Kaned, Y. (2002) Geophys. Res. Lett. 29, 43-1-43-4.

23) de Moura, F. A. B. F., Tirnakli, U., and Lyra, M. L. (2000) Phys. Rev. E 62, 6361-6365.

24) Lyra, M. L., and Tsallis, C. (1998) Phys. Rev. Lett. 80, 53-56.

25) Dieterich, J. (1994) J. Geophys. Res. 99, 2601-2618.

26) Ruina, A. L. (1983) J. Geophys. Res. 88, 10359-10370. 